\title{
Analyzing single-case data with visually guided randomization tests
}

\author{
JOHN FERRON and LYNN FOSTER-JOHNSON \\ University of South Florida, Tampa, Florida
}

\begin{abstract}
The valid use of a randomization test with single-case data typically involves the choice of a test statistic prior to gathering data. In situations in which it is difficult to anticipate the form of the effect, the early specification of the test statistic can be problematic. The purpose of this paper is to demonstrate methods that will allow single-case researchers to conduct valid randomization tests in situations in which they wish to delay the specification of the test statistic until the data have been observed. A concrete example is given to motivate the use, explicate the logic, and demonstrate the conduct of this method.
\end{abstract}

Single-case designs have been employed extensively by behavior analysts in their investigations of human conduct. Behavior is conceptualized as a consequence of interactions between individuals and their environments. To understand the phenomenon, repeated measures of the behavior are taken, while exposing the individual to all levels of the treatment. Experimental control over the behavior is demonstrated when changes in the dependent variable are clearly a function of changes in the treatment or independent variable. The nature of single-case research lends itself well to applied settings, making it an increasingly popular research method within psychology and education. As the use of single-case designs has spread, the discussion of methodological issues has increased (Kratochwill, 1992).

Among the issues receiving attention is the analysis of single-case data. The most common form of analysis is visual inspection, in which the data are presented in graphic form, allowing the analyst to reach conclusions about the effect of the treatment by visual scrutiny. The conclusions and interpretations are based on the interactions among the form of the anticipated effect, the degree to which the data appear to fit this form, and the total context from which the data come (Parsonson \& Baer, 1992). Visual analysis is easy to use, widely accessible, extremely flexible, and encourages a minimum transformation of the data-advantages that lend themselves well to the applied researcher.

Visual analysis has been presented as a conservative technique that is only sensitive to large effects (Baer, 1977; Parsonson \& Baer, 1986). The resulting assumption was that Type II errors were likely to result when effects were small but that few Type I errors would be made. However,

The authors thank Patrick Onghena and three anonymous reviewers for comments on an earlier version of this manuscript. L.F.-J. is currently a Faculty Research Statistics Specialist at the Amos Tuck School of Business, Dartmouth College. Correspondence concerning this article should be addressed to J. Ferron, Department of Educational Measurement and Research, University of South Florida, 4202 East Fowler Ave.FAO 100U, Tampa, FL 33620 (e-mail: ferron@tempest.coedu.usf.edu). the assumption of few Type I errors has been challenged. Matyas and Greenwood (1990) found that, in the presence of positive autocorrelation, judges tended to falsely conclude that treatment effects were present. Stocks and Williams (1995) also found inflated Type I error rates, especially in data with trends. Furthermore, the degree of inflation increases when one takes into account the coupling of response-guided experimentation with visual inspection (Allison, Franklin, \& Heshka, 1992), two practices that are common in single-case research. These results, combined with the low level of agreement among visual analysts (DeProspero \& Cohen, 1979; Knapp, 1983; Park, Marascuilo, \& Gaylord-Ross, 1990), make sole reliance on visual analysis questionable.

Other methods of data analysis include descriptive methods, such as the positioning of celeration lines (Stocks \& Williams, 1995) and effect sizes (Kromrey \& Foster-Johnson, 1996), as well as statistical methods that result in probability statements, such as traditional $t$ and $F$ tests, interrupted time-series analysis (McCleary \& Welsh, 1992), and randomization tests (Edgington, 1992). Whether one advocates using a statistical analysis to supplement a visual analysis seems to depend on the degree to which probability statements are valued. There are those who maintain that probability levels are unnecessary for single-case research (Baer, 1977; Skinner, 1963). However, an advantage of statistical methods is the inclusion of probability levels in the researcher's decision about the success of the independent variable and the unbiased conveyance of this decision to the research world.

The degree to which this advantage is realized depends on the believability of the calculated probability. With single-case designs, the validity of the $p$-values resulting from some forms of statistical analyses has been questioned. This skepticism has been fueled by results from studies that have found that traditional statistics have been unable to control Type I error rates when the data are autocorrelated (Greenwood \& Matyas, 1990; Toothaker, Banz, Noble, Camp, \& Davis, 1983), and interrupted timeseries analyses have yielded questionable results when series are short (Greenwood \& Matyas, 1990). Random- 
ization tests, however, provide a valid statistical test when random assignment is incorporated into the design, the randomization distribution is based on data divisions consistent with the random assignment procedure, and the test statistic is specified prior to observing the data (Edgington, 1980b).

\section{Randomization Tests}

To conduct a statistically valid randomization test, a single-case researcher would plan a design that incorporated some form of randomization. For example, the researcher could randomly assign Condition B to three of six time blocks. The researcher would then select a test statistic on the basis of the anticipated effect of the independent variable. For example, the researcher may hypothesize that there is a difference between means for Condition $\mathrm{B}$ and Condition $\mathrm{A}$. The researcher would then conduct the study and calculate the test statistic for the observed data. This would result in an obtained test statistic value. The test statistic would then be calculated for all combinations of the data that could have resulted from the randomization procedure, thereby creating a randomization distribution. If the researcher randomly assigns Condition $B$ to three of six time blocks, there are $20(6 ! /(3 ! 3 !))$ possible assignments and, thus, 20 values in the randomization distribution. Statistical significance is then determined by comparing the obtained test statistic to the randomization distribution.

Although randomization tests conducted in this manner have the advantage of being able to control Type I error rates, their use has not been accepted without reservations--particularly for professionals conducting research in applied settings. One concern among applied researchers focuses on the need for the researcher to incorporate some form of randomization in the study (Kazdin, 1980; Matyas \& Greenwood, 1991). This requirement is the antithesis to what would be done in a typical responseguided experiment, and determining treatment assignments randomly could potentially produce a treatment sequence that is ethically or practically unacceptable. A1though this issue has not been completely resolved, the number of methods for incorporating randomization has increased, making it more likely that researchers will be able to choose a randomization method that is sensitive to clinical and ethical requirements.

Currently, a wide variety of randomization methods have been proposed. For phase designs, interventions can be assigned randomly (see, e.g., Edgington, 1995; Onghena, 1992), or treatments can be assigned randomly to blocks of time (see, e.g., Edgington, 1995; Levin, Marascuilo, \& Hubert, 1978). For designs in which the treatments should be alternated rapidly, both completely randomized (Edgington, 1995) and restricted randomization schemes have been proposed (Onghena \& Edgington, 1994). For designs involving multiple baselines, methods include randomly assigning individuals to predefined baselines (Wampold \& Worsham, 1986), randomly assigning intervention points within each baseline (Marascuilo \& Busk, 1988), and randomly assigning individuals to baselines with randomly determined interventions (Koehler \& Levin, 1996). In addition, methods have been proposed that allow the design to be partially responsive to the emerging data (Edgington, 1980a; Ferron \& Ware, 1994).

\section{Concern With Predefined Test Statistics}

A second concern with the application of randomization tests centers around the need to determine a test statistic prior to looking at the data. In single-case research, a large variety of treatment effects may result, which is evidenced by the fact that visual analysts attend to many different characteristics of graphic data when making decisions about the success of the treatment. The attributes to be considered include the stability of the data within a condition, the variability of the data within and between phases, the degree data overlap between phases, the number of data points within phases, changes in trends within and between phases, changes in level between phases, the similarity of patterns across phases, and the overall pattern of the data (Parsonson \& Baer, 1986). The large number of potential effects has led to the enumeration of a variety of test statistics (see, e.g., Edgington, 1980b; Wampold \& Worsham, 1986). Choosing the most sensitive test statistic requires the researcher to correctly anticipate the nature of the treatment effect. Thus, the usefulness of randomization tests depends on the researcher's ability to select an appropriate test statistic (Ferron, 1993; Matyas \& Greenwood, 1991).

A researcher who uses a randomization test with singlecase data runs the risk of missing visually obvious effects because the predefined test statistic is not consistent with the actual effect. To illustrate this potential problem, consider a researcher who anticipates that the treatment will lead to abrupt changes in level, as is depicted in the top panel of Figure 1, but who finds that the changes are more gradual, resulting in trend shifts, as is displayed in the bottom panel of Figure 1. A test statistic based on the difference between phase means would make sense for the anticipated effect but would be relatively insensitive to the actual effect.

Researchers may be tempted to simply select a test statistic after viewing the data, but such a strategy would invalidate the statistical test. Because the researchers know which treatments were associated with which phases, they could pick a test statistic that capitalized on chance fluctuations in the data. They would essentially be considering many possible tests but making no adjustment for the fact that many tests were being considered. Type I error rates could be controlled if the researchers enumerated a set of test statistics prior to gathering data and then conducted a randomization test with each test statistic, using a Bonferroni correction. Although this method allows mul- 

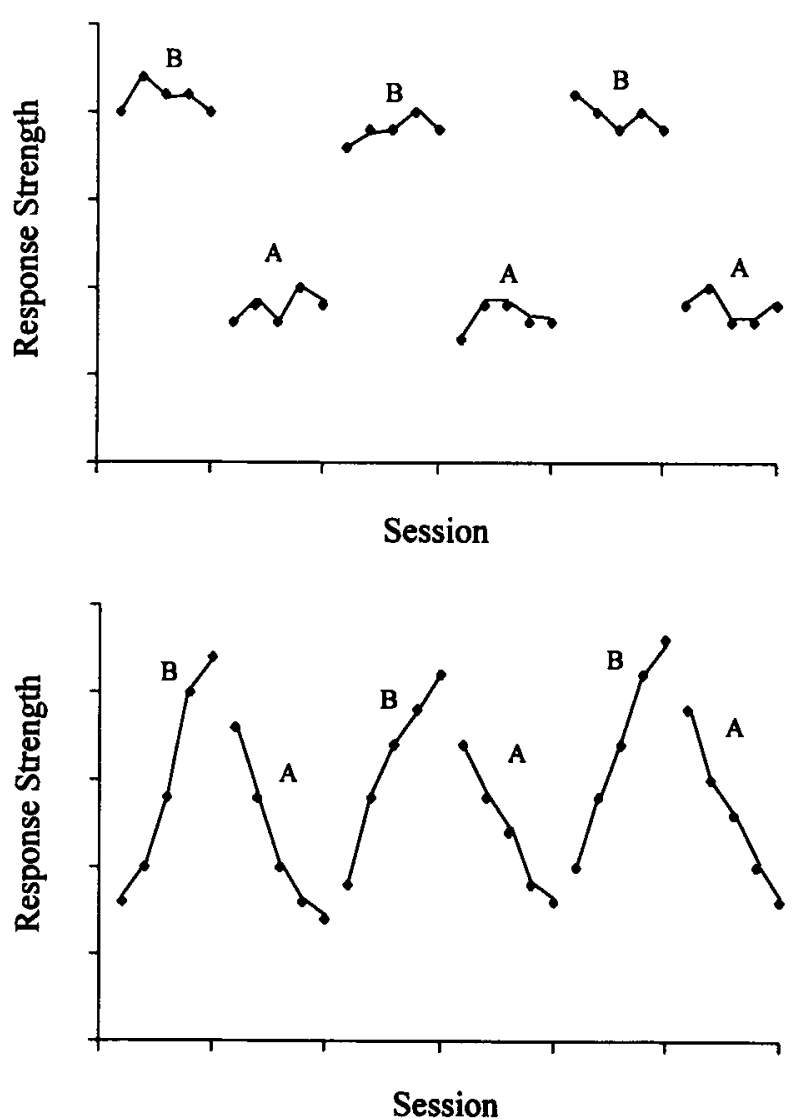

Figure 1. A treatment effect showing an abrupt change in level (top), and a treatment effect showing a change in trend (bottom).

tiple specific tests to be considered, the power for any one of the specific tests would be lower than it would have been if it had been the only test conducted.

The issue of test statistic specification is essentially a concern with optimizing power. Several studies have empirically examined the power associated with randomization tests (Ferron \& Onghena, 1996; Ferron \& Ware, 1995; Onghena, 1994). These studies found the power of randomization tests to be lacking in many situations. Consequently, attempts to optimize power by carefully specifying the test statistic appear justified.

The purpose of this article is to present a modification to the traditional randomization test procedure that allows researchers to delay the specification of a test statistic until more information about the form of the effect is obtained. These modifications will be valuable to single-case researchers who prefer to conduct an analysis that controls the Type I error rate but who study effects that are difficult to specify in advance.

\section{DELAYING THE CHOICE OF A TEST STATISTIC}

\section{General Modifications}

To delay the specification of the test statistic, the randomization procedure must be modified to include indi- viduals who understand the randomization scheme but are unaware of the actual treatment assignment. These individuals would be able to divide the observations into all of the possible data divisions but would not be informed of which division corresponded to the actual experiment. They would work as a team of visual analysts, observing graphical displays of the data, exploring different test statistic possibilities, and eventually recommending a test statistic.

The details of how the visual analysts are worked into the experimental scheme are outlined in the following set of steps: (1) the researcher plans a study that incorporates some form of randomization; (2) the researcher conducts the study; (3) the researcher constructs a data table that contains observation times, the corresponding measurements of the dependent variable, but no information about which treatment was associated with each observation time; (4) visual analysts who are naive to the actual treatment sequence are given the data table and information on the set of treatment sequences made possible by the randomization scheme; (5) the visual analysts explore the various data divisions graphically and by calculating possible test statistic values; (6) the visual analysts suggest an appropriate test statistic; (7) the researcher calculates the obtained test statistic value on the basis of the actual treatment sequence; $(8)$ the researcher then forms a randomization distribution by calculating the test statistic for all treatment sequences that could have resulted from the randomization procedure; and (9) statistical significance is determined by comparing the obtained test statistic with the randomization distribution. When these steps are compared with those for a traditional randomization test, two differences become apparent: The test statistic is defined after the data are viewed, and visual analysts who are naive to the actual treatment assignment are utilized. The modification can be described more fully by turning to a concrete example.

\section{Illustrative Example}

Consider a researcher who wishes to examine the effect of a videotaped self-monitoring program on a student's social interactions. The researcher hypothesizes that the videotaped self-monitoring program (Treatment B) will produce higher levels of appropriate social interactions than the current treatment (Treatment A), which incorporates a strict reinforcement schedule. The researcher determines that a study with approximately 30 observation points (sessions) will be of sufficient length to ascertain the effectiveness of the treatment. The researcher now decides on a randomization scheme that is feasible for the experiment. The researcher decides to randomly assign treatments to time blocks. This scheme is frequently described in the randomization test literature (see, e.g., Edgington, 1995; Levin et al., 1978), and randomization tests based on this scheme have reasonable levels of power when the treatment effect is correctly specified (Ferron \& Onghena, 1996). The 30 sessions are then divided into six equal length time blocks. Treatment B, the videotaped self-monitoring program, is randomly as- 
signed to three of the six time blocks, and Treatment A is assigned to the remaining three blocks. The researcher then conducts the study.

After the study, the researcher makes a data table containing two columns of numbers. The first column contains the numbers 1 through 30 , which index the 30 sessions. The second column contains the corresponding social interaction measurements. Note that the data table contains no information about which treatments correspond with which measurements. The data table is then shared with a team of visual analysts who are familiar with the purpose of the study, the measurement of the social interactions, the videotaped self-monitoring program, and the randomization method, but naive to the actual treatment assignment. The visual analysts have the task of recommending a test statistic to be used in the randomization test.

Since the self-monitoring program is expected to increase the amount of social interaction, the visual analysts reason that the mean level of interaction in the B blocks should be higher than the mean level of interaction in the A blocks. This leads to consideration of a common directional test statistic, the difference between treatment means, $T_{1}=\bar{X}_{\mathrm{B}}-\bar{X}_{\mathrm{A}}$. The empirical distribution for this test statistic is found by calculating its value for each of the 20 data divisions. The 20 values are ranked and displayed in Figure 2, along with the visual display corresponding to each data division. The test statistic is maximized for Division 1, AAABBB. The visual display for this division troubles the analysts. There is a notable increase in social interaction during the beginning of the A phase that is difficult to explain and a sharp decline in social interaction at the end of the B phase that is difficult to explain. Thus, even though the mean level of social interaction is 10.7 points higher in the $\mathrm{B}$ phase, the trends within the phases are not consistent with expectations.

After looking at the graphic display of the data, the visual analysts decide that it may be more appropriate to consider a test statistic that takes into account the trends in the data. The analysts calculate the slope, $b$ for each of the six blocks of data, then average the three slopes corresponding to the $\mathrm{B}$ blocks and the three slopes corresponding to the A blocks. A directional test statistic is found by taking the difference between the average slopes, $T_{2}=\bar{b}_{\mathrm{B}}-\bar{b}_{\mathrm{A}}$. This statistic is maximized for division 16. Again the analysts turn to the visual display of the data. The level of social interaction increased during the two $B$ phases and decreased during the two A phases, as expected.

A more detailed visual inspection reveals some inconsistencies with expectations. The first six points of the second B phase show little to no increase in social interactions. This length of a delay is not expected and is not consistent with the change observed across the first $B$ phase. Why is there a lengthy delay before the treatment takes hold? If an explanation can be found, why is the delay only observed in the second B phase? A similar difficulty arises in trying to explain the pattern within the second A phase. There is little to no drop in social interactions during the first five observations. This delay is not expected and is inconsistent with the immediate drop in social interactions that accompanied the first A phase. A second test statistic has been considered, and again difficulties have arisen when the visual analysts looked toward the more subtle changes taking place within a phase.

Uncomfortable with the two relatively common test statistic choices, the visual analysts decide to entertain a less conventional test statistic. The graphical display of the data leads the analysts to believe that there is a change in the level of social interaction. This change, however, does not appear to be abrupt but rather appears to take place over several observations, suggesting that skill acquisition is occurring in later stages of the condition. As a result, the analysts decide to look at a change in level, but one where the observations later in a time block are weighted more heavily. For each time block, the analysts weight the observations on the basis of their position within the block. The first observation is multiplied by one, the second by two, the third by three, the fourth by four, and the fifth by five. The weighted observations are then summed and divided by the sum of the weights, 15 . This yields an average for each block that is more heavily influenced by the observations occurring later in the block. The statistics are then averaged for the three B blocks and averaged for the three A blocks. The third test statistic, also directional, is defined as the difference between the means of these weighted averages. This can be represented more concisely as $T_{3}=\sum w X_{\mathrm{B}}-\sum w X_{\mathrm{A}}$, where $w$ is defined as the observation number within the time block divided by the sum of the observation numbers. The sum of the observation numbers will be 45 ( 15 per block times three blocks). The values for this test statistic are also displayed in Figure 2.

The third test statistic is maximized for Division 2. A more detailed analysis of the graphic representation for Division 2 reveals results that are consistent with treatment expectations. The first $B$ phase shows an increase in social interactions. The first A phase shows a drop, followed by stabilization at a relatively low level. The second B phase results in the expected increase in social interactions, followed by stabilization at a relatively high level. Finally, the second A phase leads to a drop in social interactions. After viewing the graphs for each of the 20 possible data divisions, the visual analysts are even more convinced that Division 2, corresponds to the treatment sequence that was actually used. Consequently, the visual analysts recommend that the third test statistic be used in the randomization test.

The researcher then conducts the randomization test. Since the treatment sequence corresponding to Division 2 was the one that was actually used, the obtained test statistic value was 10.9. This was the largest value in the empirical distribution, resulting in the smallest possible $p$ value for this experiment, a $p$ value of .05 . 
Division 1 - AAABBB

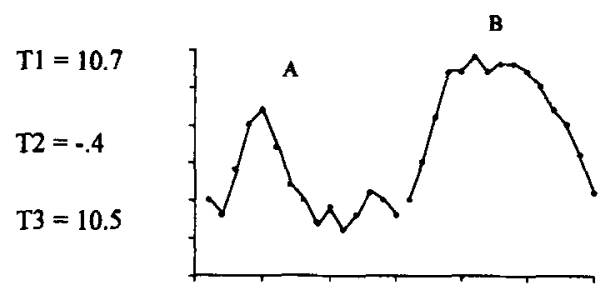

Division 3 - BAAABB

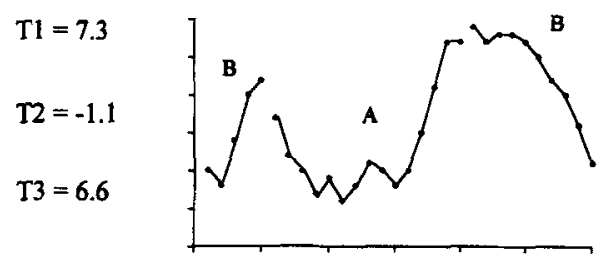

Division 5 - ABAABB

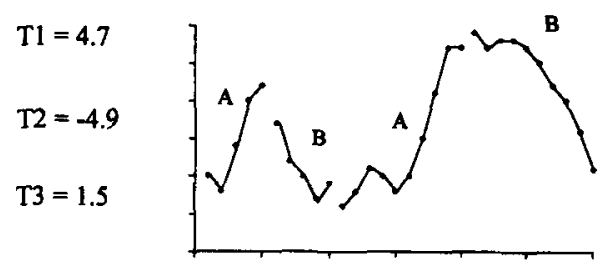

Division 7 - AABABB

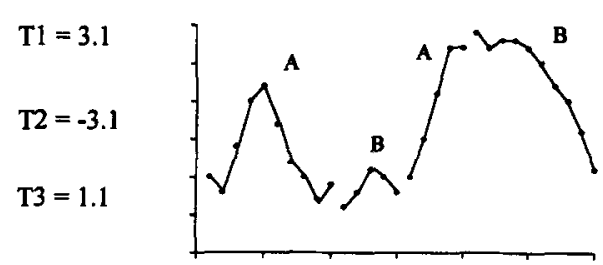

Division 9 - BBAABA

$$
\begin{aligned}
& \mathrm{T} 1=2.1 \\
& \mathrm{~T} 2=-.2 \\
& \mathrm{~T} 3=1.9
\end{aligned}
$$

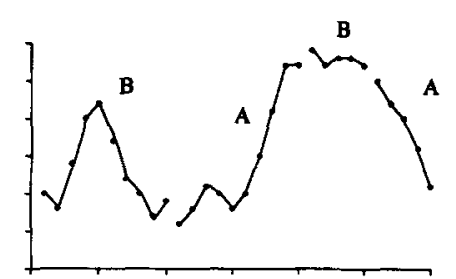

Division 2 - BAABBA
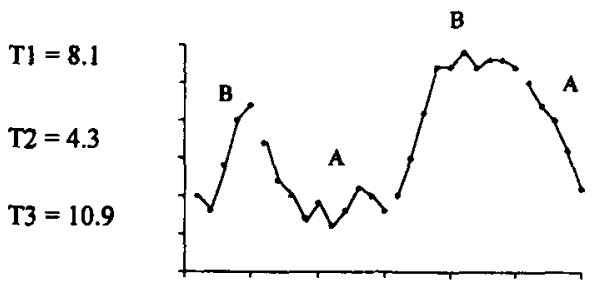

Division 4 - ABABBA
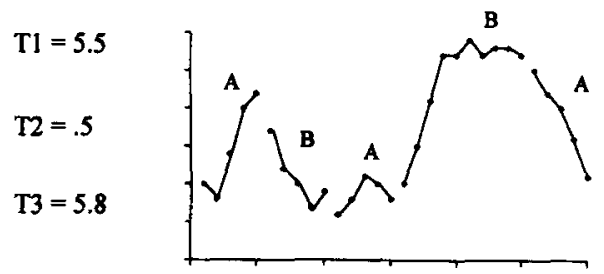

Division 6 - AABBBA

$$
\begin{aligned}
& \mathrm{T} 1=3.9 \\
& \mathrm{~T} 2=2.3 \\
& \mathrm{~T} 3=5.4
\end{aligned}
$$

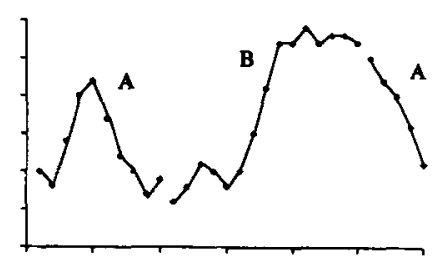

Division 8 - BAABAB
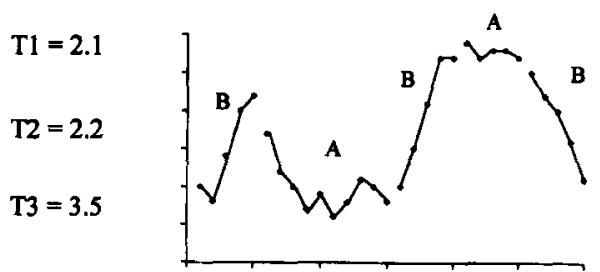

Division 10 - BABABA
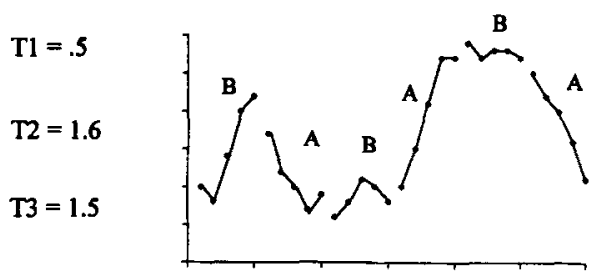

Figure 2. Visual display and test statistics for each of the 20 possible data divisions, $T_{1}=\bar{X}_{\mathrm{B}}-\bar{X}_{\mathrm{A}}, T_{2}=$ $\bar{b}_{\mathrm{B}}-b_{\mathrm{A}}, T_{3}=\sum w X_{\mathrm{B}}-\sum w X_{\mathrm{A}}$, where $w$ is defined as the observation number within the time block divided by the sum of the observation numbers.

\section{DISCUSSION}

\section{Validity of the Test}

One may question how the $p$ value can be trusted with such an interactive method of data analysis. Under some conditions, looking at the data prior to choosing a test statistic will invalidate the statistical test. For example, if the researcher who conducted the study looked at the data and then chose a test statistic, the validity of the randomization test would be compromised. The problem 
Division 11 - ABABAB

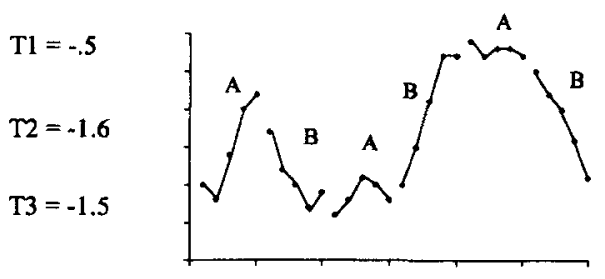

Division 13 - ABBABA

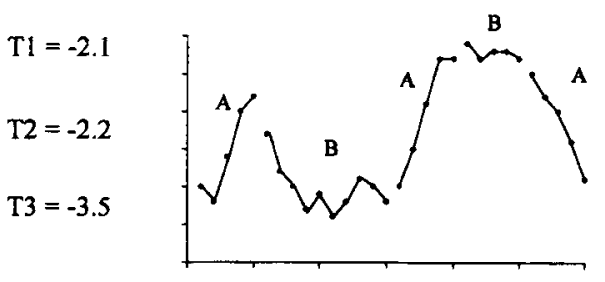

Division 15 - BBAAAB

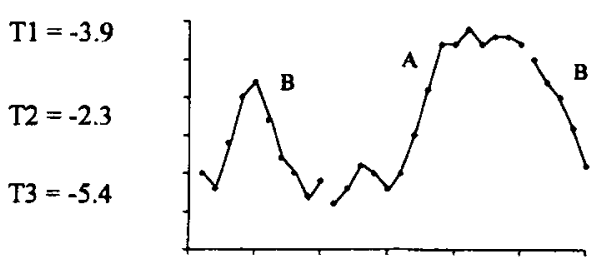

Division 17 - BABAAB

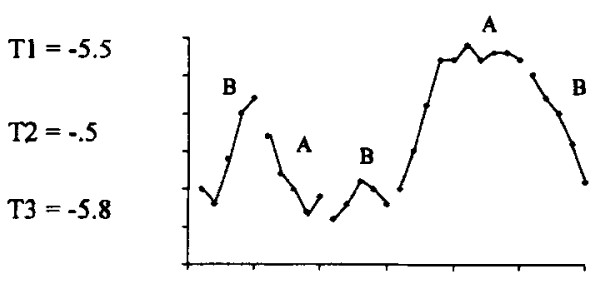

Division 19 - ABBAAB

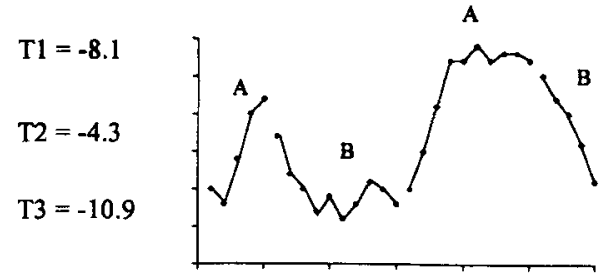

Division 12 - AABBAB
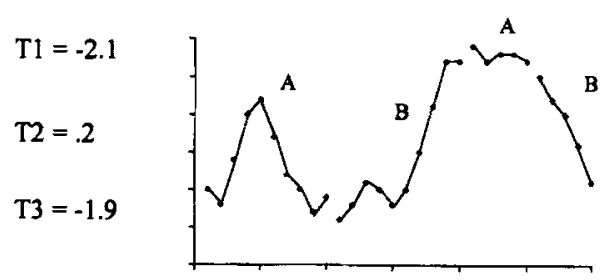

Division 14 - BBABAA

$\mathrm{T} 1=-3.1$
$\mathrm{~T} 2=3.1$
$\mathrm{~T} 3=-1.1$

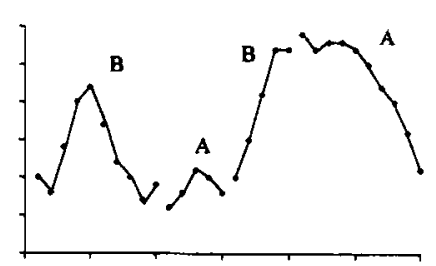

Division 16 - BABBAA

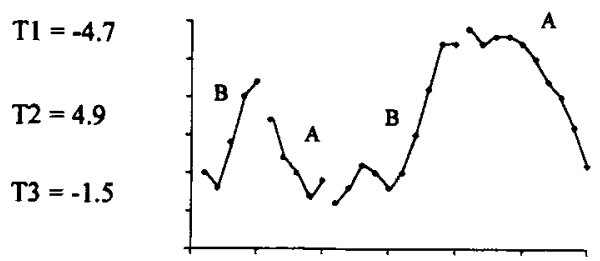

Division 18 - ABBBAA

$T 1=-7.3$
$T 2=1.1$
$T 3=-6.6$

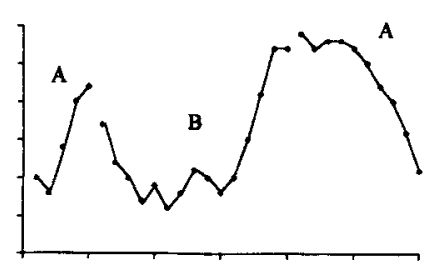

Division 20 - BBBAAA
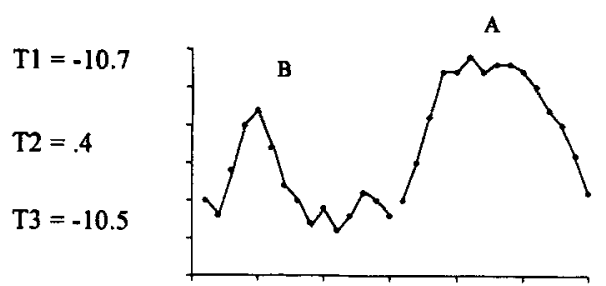

Figure 2 (Continued).

arises because the researcher knows the actual treatment assignment and can capitalize on chance differences in the observed data. This problem is circumvented in the modified randomization test by incorporating visual analysts who are blind to the actual treatment assignment.

To more fully explicate the legitimacy of the calculated $p$-value, it may be helpful to review the validity argument used for the more traditional randomization test. For a directional test, the $p$-value is defined as the probability of obtaining a test statistic value as large as or larger than the one obtained, if there is no difference in the effectiveness of the treatments. If there is indeed no difference in the effectiveness of the treatments, all of the possible treatment assignments will produce exactly the 
same set of data. Thus, the same randomization distribution results from any of the treatment assignments. Five percent of the possible treatment assignments will lead to test statistics in the upper $5 \%$ of the randomization distribution. Since the actual assignment is chosen randomly, each of the possible assignments has the same probability of being chosen. Thus, the probability of obtaining a test statistic value in the upper $5 \%$ of the randomization distribution is .05.

For the modified randomization test with the delay in the specification of the test statistic, the logic is similar. If there is no difference in the treatments, the same data will result from any of the possible assignments. The data table given to the visual analysts would be exactly the same for any of the treatment assignments. Thus, the visual analysts would engage in the same exploration and come to the same test statistic recommendation for any of the possible treatment assignments. This implies that the same randomization distribution would be formed regardless of the treatment assignment. Consequently, $\alpha \times 100 \%$ of the possible treatment assignments will lead to test statistics in the upper $\alpha \times 100 \%$ of the randomization distribution. Since each of the possible assignments has the same probability of being chosen, the probability of choosing an assignment that leads to an obtained test statistic in the upper $\alpha \times 100 \%$ of the randomization distribution is $\alpha$.

\section{Constructing Graphs for All Possible Treatment Assignments}

A second issue of concern centers around whether it is practically feasible for the visual analysts to construct graphs for all the possible treatment assignments. For the above example, there were 20 possible assignments, and all 20 graphs were constructed. The task will become quite burdensome as the number of possible assignments increases, and randomization schemes have been proposed that have hundreds and sometime thousands of different data divisions. Fortunately, the visual analysts do not need to construct a graph for each data division.

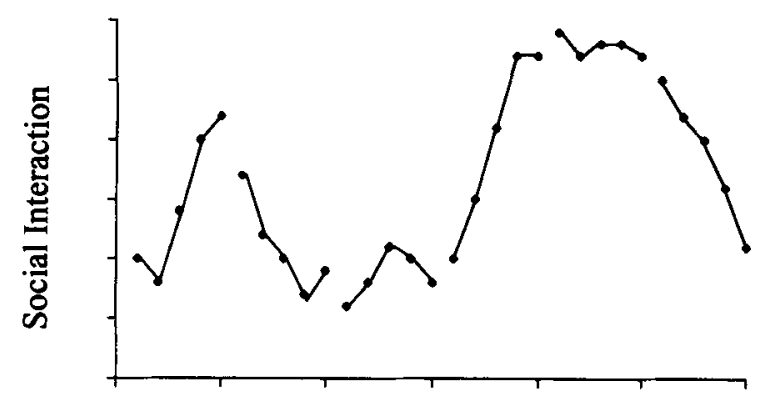

Session

Figure 3. Visual display that does not indicate the actual treatment assignment.
A single graph that does not contain information about the treatment assignment can be constructed that will be sufficient for this approach. As an example, consider the graph in Figure 3, which corresponds to the data in the social interaction example.

Visual analysts looking at these data may reason that videotaped self-monitoring was used during the first block of time, leading to an expected increase in social interaction. The second block of time shows a pronounced decrease in social interaction, which would make sense if the treatment switched back to the strict reinforcement schedule. The interaction does not continue to drop but stays low during the third block. This pattern is explainable if the same treatment was continued. There is a sharp increase in social interaction during the fourth time block, which could be explained if the videotaped selfmonitoring program was reinstated. The level of interaction remains at a high level during the fifth block, suggesting a continuation of the videotaped self-monitoring. Finally, the last time block shows a drop in interaction, which can be explained if there was a switch back to the reinforcement treatment. After this general sort of analysis, the visual analysts can consider test statistics that would be sensitive to the perceived effect.

\section{Conclusions}

The issue of how best to design and analyze the data from single-case studies continues to be discussed. Although a great deal of methodological research has been done, the evidence has not led to the emergence of a single optimal method. For those who place a premium on design flexibility, the responsive design, coupled with a visual analysis, remains an attractive option. For researchers who are more interested in controlling Type I error rates and ruling out alternative explanations through random assignment, an attractive alternative involves the use of a partially random design, coupled with a randomization test. If the researcher chooses the latter route, other decisions must be made. The researcher must determine how random assignment will be incorporated into the design and how the test statistic will be chosen.

Both the traditional randomization test method, which involves specifying the test statistic in advance, and the visually guided method control the Type I error rate. In addition, they both require a subjective decision about what test statistic to use. The difference between the strategies lies in the information used in making the decision. With a traditional randomization test, the decision is based on theory and/or past research. When these sources of information are sufficient to define the treatment effect, there is no reason to modify the traditional randomization test strategy. When these sources of information are not sufficient, researchers may wish to obtain more information to inform their decision. The visually guided randomization test allows additional information to be obtained by viewing the data on the outcome variable. When 
there is a treatment effect, the outcome data will tend to contain relevant information about the form of the effect. The use of this information would tend to increase the likelihood that an appropriate test statistic would be specified, which would increase the chances of detecting real differences.

Although the visually guided strategy allows additional information to be obtained, it does not guarantee that the most appropriate test statistic will be chosen. The outcome data will contain noise that may lead the visual analysts to make wrong decisions about when phase changes occurred and which test statistic to use. The degree to which reasonable decisions will be made will be partly dependent on the visual analysts' methodological skills and their understanding of the treatment and behavior under investigation. Consequently, the choice of visual analysts should be made judiciously.

It is not being suggested that visually guided randomization tests become the method for analyzing singlecase data. There clearly is not evidence to support such a claim. Rather, the method is being presented as an additional analysis option to be considered in the conduct of single-case research. The method is unique in that it allows Type I error rates to be controlled, without requiring a test statistic to be specified prior to data collection. This set of characteristics makes the method useful in situations in which the researcher places a high priority on controlling the Type I error rate but is uncomfortable specifying a test statistic prior to collecting data.

Future research should examine the power associated with both traditional and visually guided randomization tests, as well as the power of other analysis options. Past research aimed at addressing the power of randomization tests has assumed that the test statistic was appropriately identified (Ferron \& Onghena, 1996; Ferron \& Ware, 1995; Onghena, 1994). Future research needs to expand this work by considering situations in which there is uncertainty concerning the form of the treatment effect. It is in these situations that it would be particularly interesting to explore the power of both the traditional and visually guided strategies. A second line of research should examine the degree to which the number of individuals involved in the test statistic decision alters the probability of test statistic misspecification in both traditional and visually guided randomization tests.

\section{REFERENCES}

Allison, D. B., Franklin, R. D., \& HeshKa, S. (1992). Reflections on visual inspection, response guided experimentation, and Type I error rate in single-case designs. Journal of Experimental Education, 61, 45-51.

BAER, D. M. (1977). Perhaps it would be better not to know everything. Journal of Applied Behavior Analysis, 10, 167-172.

DeProspero, A., \& COHEN, S. (1979). Inconsistent visual analysis of intrasubject data. Journal of Applied Behavioral Analysis, 12, 573-579.

EDGINGTON, E. S. (1980a). Overcoming obstacles to single-subject experimentation. Journal of Educational Statistics, 5, 261-267.

EDGINGTON, E.S. (1980b). Validity of tandomization tests for one-subject experiments. Journal of Educational Statistics, 5, 235-251.
Edgington, E. S. (1992). Nonparametric tests for single-case experiments. In T. R. Kratochwill \& J. R. Levin (Eds.), Single-case research design and analysis: New directions for psychology and education (pp. 133-157). Hillsdale, NJ: Erlbaum.

EDGINGTON, E. S. (1995). Randomization tests (3rd ed.). New York: Marcel Dekker.

FERron, J. (1993, April). Suggested solutions to problems facing the use of randomization tests with single-case designs. Paper presented at the annual meeting of the American Educational Research Association, Atlanta.

Ferron, J., \& Onghena, P. (1996). The power of randomization tests for single-case phase designs. Journal of Experimental Education, 64, 231-239.

FERRON, J., \& WARE, W, (1994). Using randomization tests with responsive single-case designs. Behavior Research \& Therapy, 32, 787-791.

FERRON, J., \& WARE, W. (1995). Analyzing single-case data: The power of randomization tests. Journal of Experimental Education, 63, 167-178.

Greenwood, K. M., \& Matyas, T. A. (1990). Problems with the application of interrupted time series analysis for brief single-subject data. Behavioral Assessment, 12, 355-370.

KAZDIN, A. E. (1980). Obstacles in using randomization tests in singlecase experimentation. Journal of Educational Statistics, 5, 253-260.

KNAPP, T. J. (1983). Behavior analysts' visual appraisal of behavior change in graphic display. Behavioral Assessment, 5, 155-164.

KoEHLER, M. J., \& LEVIN, J. R. (1996, April). A sharper analytical tool for the multiple-baseline design. Paper presented at the annual meeting of the American Educational Research Association, New York.

KraTOCHWILL, T. R. (1992). Single-case research design and analysis: An overview. In T. R. Kratochwill \& J. R. Levin (Eds.), Single-case research design and analysis: New directions for psychology and education (pp. 1-14). Hillsdale, NJ: Erlbaum.

Kromrey, J. D., \& Foster-Johnson, L. (1996). Determining the efficacy of intervention: The use of effect sizes for data analysis in singlesubject research. Journal of Experimental Education, 65, 73-93.

Levin, J. R., Marascuilo, L. A., \& Hubert, L. J. (1978). N = nonparametric randomization tests. In T. R. Kratochwill (Ed.), Single subject research: Strategies for evaluating change (pp. 167-196). New York: Academic Press.

Marascullo, L. A., \& Busk, P. L. (1988). Combining statistics for multiple-baseline $\mathrm{AB}$ and replicated $\mathrm{ABAB}$ designs across subjects Behavioral Assessment, 10, l-28.

Matyas, T. A., \& GREenwood, K. M. (1990). Visual analysis of singlecase time series: Effects of variability, serial dependence, and magnitude of intervention effects. Journal of Applied Behavior Analysis, 23, 341-351.

Matyas, T. A., \& Greenwood, K. M. (1991). Problems in the estimation of autocorrelation in brief time series and some implications for behavioral data. Behavioral Assessment, 13, 137-157.

MCCleary, R., \& Welsh, W. N. (1992). Philosophical and statistical foundations of time-series experiments. In T. R. Kratochwill \& J. R. Levin (Eds.), Single-case research design and analysis: New directions for psychology and education (pp. 41-91). Hillsdale, NJ: Erlbaum.

ONGHENA, P. (1992). Randomization tests for extensions and variations of ABAB single-case experimental designs: A rejoinder. Behavioral Assessment, 14, 153-171.

ONGHENA, P. (1994). The power of randomization tests for single-case designs. Unpublished doctoral dissertation, Katholieke Universiteit Leuven.

Onghena, P., \& Edgington, E. S. (1994). Randomization tests for restricted alternating treatments designs. Behavior Research \& Therapy, 32, 783-786.

Park, H. S., Marascuilo, L., \& Gaylord-Ross, R. (1990). Visual inspection and statistical analysis in single-case designs. Journal of $E x-$ perimental Education, $\mathbf{5 8}, 311-320$.

Parsonson, B. S., \& BaEr, D. M. (1986). The graphic analysis of data. In A. Poling \& W. R. Fuqua (Eds.), Research methods in applied behavior analysis: Issues and advances (pp. 157-186). New York: Plenum.

Parsonson, B. S., \& BaER, D. M. (1992). The visual analysis of data, and current research into the stimuli controlling it. In T. R. Kratoch- 
will \& J. R. Levin (Eds.), Single-case research design and analysis: New directions for psychology and education (pp. 15-40). Hillsdale, $\mathrm{NJ}$ : Erlbaum.

SkINNER, B. F. (1963). Operant behavior. American Psychologist, 18, 503-515.

STOCKS, J. T., \& Williams, M. (1995). Evaluation of single subject data using statistical hypothesis tests versus visual inspection of charts with and without celeration lines. Journal of Social Research, $\mathbf{2 0}$, 105-126.
Toothaker, L. E., Banz, M., Noble, C., Camp, J., \& Davis, D. (1983). $\mathrm{N}=1$ designs: The failure of ANOVA-based tests. Journal of Educational Statistics, 8, 289-309.

WAMPOLD, B. E., \& Worsham, N. L. (1986). Randomization tests for multiple-baseline designs. Behavioral Assessment, 8, 135-143.

(Manuscript received July 17, 1995 revision accepted for publication June 5, 1997.) 